\title{
Dynamic modeling and simulation of a heat pump system for enhancing cycle flexibility
}

\author{
Yutaka Watanabe ${ }^{1,{ }^{*}}$ and Alberto Traverso ${ }^{2}$ \\ ${ }^{1}$ Central Research Institute of Electric Power Industry, Energy Engineering Research Laboratory, 2-6- \\ 1 Ngasaka, Yokosuka-shi, Kanagawa 249-0196, Japan \\ ${ }^{2}$ University of Genoa, Department of Mechanical Engineering, Thermochemical Power Group, Via \\ Montallegro,1, 16145 Genova, Italy
}

\begin{abstract}
This paper presents a dynamic simulation of a heat pump using butane refrigerant for the purpose of integration with a combined cycle power system and thermal energy storage to enhance flexibility. A dynamic model of a heat pump system is developed using the Amesim software and the simulation result in the case of changing the heat source and heat sink conditions is shown to validate the simulation performance.
\end{abstract}

\section{Introduction}

The fluctuating power output of renewable energy sources makes the electric grid potentially unstable, therefore it is necessary to increase the flexibility of the grid and its possibility to store energy. The PUMP-HEAT project [1] aims to develop a new advanced energy system that integrates a combined cycle system with highly efficient fast-cycling heat pumps, where thermal energy storage is used as an electrical-equivalent storage enhancing flexibility. Dynamic simulation plays an important role in the investigation of the PUMP-HEAT conceptual design and optimization under various dynamic operational conditions. In this study, a dynamic model of a heat pump system using butane was developed using Amesim [2], and the simulation example in the case of changing water flow rate of heat source and heat sink was presented to validate the simulation performance.

\section{Configuration}

Fig. 1 presents a schematic of the target heat pump system. The apparatus includes a compressor, condensers (including a desuperheater, condenser, and subcooler), a superheater, an expansion valve (or an expander in the case of PUMP-HEAT laboratory under construction at University of Genoa) and an evaporator. The heat pump system is designed to raise the temperature of hot water for district heating networks from 70 to 120 $\operatorname{deg} C$ and the refrigerant in the heat pump system is butane. The heat exchange fluid of the heat source and heat sink side is water. Operational inputs of the system are basically water conditions of heat sink and heat source side and rotary speed of a compressor.

\footnotetext{
*Corresponding author: yutaka@criepi.denken.or.jp
} 


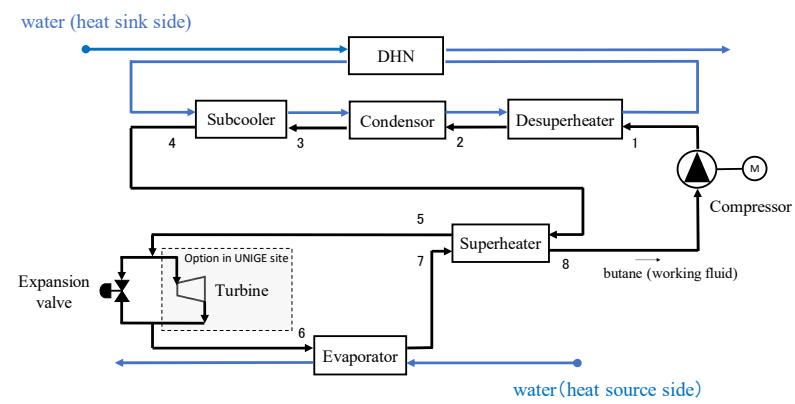

(a) Heat pump system

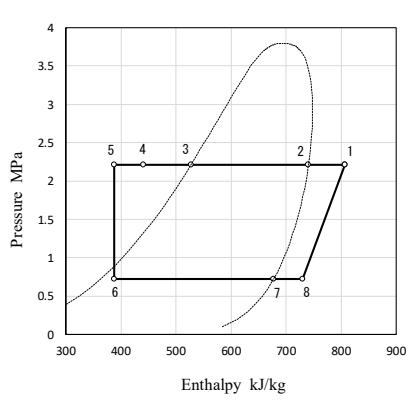

(b) P-h graph

Fig. 1. Schematic of target heat pump system

\section{Simulation}

The simulation model was constructed using Amesim. Fig.2 shows the simulation result examples in the case that the flow rate of water in both heat sink and heat source side are decreased as a step change at $500 \mathrm{sec}$. The simplification of the model and inaccuracy of setting configuration parameters caused the steady-state errors, but the dynamic simulation could be performed well using Amesim. It should be noted that setting detailed design data parameters and operational data are necessary for validating dynamic behaviour especially.
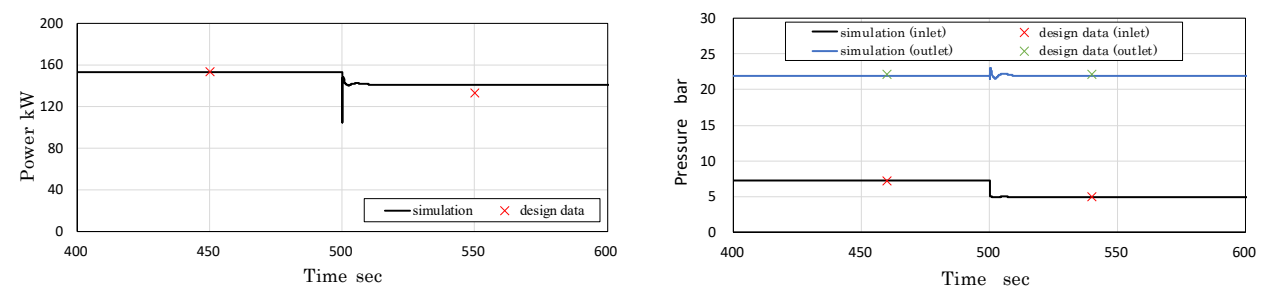

Fig. 2. Simulation example (left: compressor power, right: pressure of compressor inlet and outlet)

\section{Conclusion}

This study presented a dynamic simulation of a heat pump system using butane refrigerant. In future work, we will conduct a thorough validation study comparing dynamic simulation data with operational data from the PUMP-HEAT laboratory at University of Genoa and the impact of substituting the lamination valve with a turboexpander for further energy recovery will be investigated.

This project has received funding from the European Union's Horizon 2020 research and innovation programme under grant agreement No 764706, PUMP-HEAT.

\section{References}

1. https://www.pumpheat.eu/ (last accessed 29/04/2019)

2. https://www.plm.automation.siemens.com/global/ja/products/simcenter/simcenteramesim.html (last accessed 29/04/2019) 